\title{
The Presence and Interrater Reliability of Thoracic ALLOdYNia In A Whiplash COHORT
}

\author{
Shannon L. Bock, MMS, PA-C, CMT, Christopher J. Centeno, MD, and James M. Elliott, MSPT
}

\begin{abstract}
Background: The need exists for more definitive clinical signs indicative of abnormal central pain processing following a whiplash injury. Our observations have identified the presence of allodynia in the thoracic spine in patients with neck pain following whiplash injury. The evaluation of allodynia in the thoracic spine in a whiplash injured population may assist in the clinical diagnosis of patients believed to be suffering from central nervous system hypersensitivity.

Objective: To evaluate for the presence of allodynia in a cohort of patients
\end{abstract}

with whiplash injury.

Methods: Thirty-one patients with whiplash injuries were evaluated in this prospective study for the presence of allodynia in the thoracic spine. Thoracic vertebrae were marked by the initial evaluating clinician and a Wartenberg pinwheel was utilized to identify areas of hypersensitivity in the thoracic dermatomes. Patients were instructed to give no verbal clues, so only visual clues, as determined by the clinician, were assessed. A second clinician, blinded to the initial examination results, repeated the examination in the same fashion.

Results: Thoracic allodynia was identified in $70.97 \%$ of the observed population. There was a high level of agreement between observers (Kappa coefficient of agreement, $0.8039 ; 95 \% \mathrm{Cl}, 0.7465,0.8613 ; \mathrm{P}<0.05$ ). There was no predilection for a particular thoracic dermatome.

Conclusion: Thoracic allodynia was identified as a common, interrater-reliable, objective physical examination finding in this whiplash cohort.

Keywords: Allodynia, whiplash, interrater reliability, thoracic
In patients who have sustained whiplash injuries, the mechanisms involved in maintaining chronic pain are poorly understood. Whiplash-injured patients often present with multiple areas of chronic pain and mechanical allodynia (1). Allodynia refers to pain that is due to a stimulus that does not normally cause pain; there is a lowered threshold of pain.

Extensive studies (2-6) have investigated allodynia in animals. In general, these animal models suggest that injury resulting in increased sensitivity to noxious stimuli and pain after innocuous stimuli, may be caused by hypersensitivity in spinal cord neurons (central hypersensitivity).

In recent years, human studies

From: Centeno Clinic, Westminister, CO Address Correspondence:

Shannon L Bock, MMS, PA-C, CMT

1108 o Circle Point Rd, Bldg. 2, Suite 140 Westminster, $\mathrm{CO} 80020$

E-mail: sbock@centenoclinic.com

Disclaimer: There was no external funding in preparation of this manuscript.

Conflict of Interest: None

Manuscript received on 5/20/2005

Revision submitted on 6/02/2005

Accepted for publication on 6/19/2005 support the hypothesis of central nervous system hypersensitivity as a contributor to the persistent pain and allodynia experienced by whiplash patients $(1,7-9)$. However, literature that investigates allodynia specifically in a whiplash population is sparse. The aim of this prospective case series was to investigate the presence of thoracic allodynia as a physical finding in a whiplash patient cohort and to demonstrate the interrater reliability of allodynia

\section{Methods}

Thirty-one male and female patients with whiplash injuries due to motor vehicle accidents (MVA) were randomly selected from the patient population of a private physiatry/pain management practice specializing in spinal injuries. Patients selected ranged in post-MVA status from 0.25 years to 1.5 years. Based upon previous clinical examination, 20 of the 31 participants were classified as WAD 2 (whiplash associated disorder, Quebec Task Force classification system) and 11 as WAD 1. The investigational review board of Spinal Injury Foundation approved the study. Patients meeting inclusion criteria consented to participate in this observational study.

Exclusion criteria eliminated subjects who: 1) were identified as having gener- al (non-whiplash-related) neck or back pain lasting more than a few days, or pain lasting more than a few days following an injury or accident prior to their current motor vehicle accident; 2) had been diagnosed with a neurological condition at some point, experienced paraesthesia that lasted more than a few days, or had been diagnosed with a bulging, protruding or herniated disc; 3) had been hospitalized for any muscle, joint, or nerve condition, or who had any type of surgery of the joints or bones; 4) were diagnosed by a rheumatologist as having rheumatoid arthritis or any other connective tissue disorder such as systemic lupus erythematosus (SLE) or Reiter's syndrome; 5) had been treated with prescription medications for rheumatoid arthritis; 6) had a thoracic spine MRI showing significant findings such as disc herniation, spinal canal or foraminal stenosis, or facet arthropathy; 7) had been taking certain prescription medication(s) for pain, muscular spasm, or neuropathic pain that would mask or alter observational findings; or 8) were pregnant.

Using washable ink, the initial evaluating clinician numbered the patient's vertebrae, one through twelve, on the skin over the spinous processes. The patient 
was then given verbal instructions not to speak during the examination so the clinician could evaluate only visual clues. The patient was also told how to stop the examination should they wish to end their participation.

Each patient was placed in the prone position on the exam table. A "starting point" mark was made by the initial evaluating clinician at a point 2 centimeters (cm) bilaterally to the $\mathrm{T} 1$ spinous process. Beginning at the starting point mark, the clinician moved a Wartenberg pinwheel down each side of the thoracic spine with moderate rapidity. The clinician noted any visual clue indicative of allodynia (which is defined as a painful sensation in response to a normally nonpainful stimulus), including jump sign, twitch response, visible retraction, pilomotor response, or other non-verbal pain response. The pinwheel exam consisted of 3-4 trials in succession on each side, a number that ensured the clinician had properly identified the corresponding thoracic dermatome at which visual clues were present, and also ensured that these clues were present across multiple trials. The clinician then identified where an allodynia was present by marking the corresponding location on a form printed with a thoracic spine illustration labeled from 1-12 and left/right.

A second clinician, blinded to the outcome of the initial exam, repeated the same assessment within five minutes of the initial examination. Prior to the second exam, patients were given repeat instructions that they were to give no verbal clues. The second clinician conducted their exam in the same fashion as the first, beginning $2 \mathrm{~cm}$ bilateral from the midline mark and moving the pinwheel with moderate rapidity in a succession of 3-4 trials. The second clinician then completed his own thoracic spine form independent of the first clinician's. The two separate forms were then attached to patient information and placed in a folder for statistical analysis by an unbiased third party.

It is important to note that every patient was evaluated by the same initial clinician to provide continuity in marking the thoracic spinous processes and starting points. The second evaluating clinician was chosen randomly from a pool of three qualified clinicians. All clinicians have similar clinical experience and utilize a Wartenberg pinwheel exam on a routine basis in their clinic setting. Agreement between the two evaluators was assessed by determining Kappa coefficients (SAS Institute, Inc., statistical software), a statistical measure of agreement between observers

\section{RESULTS}

Of the 31 patients evaluated, the initial evaluating clinician was unable to identify evidence of allodynia on the basis of visual clues in nine of the patients, and therefore, the nine were not subsequently evaluated by a second clinician. These nine patients were excluded from statistical analysis for kappa coefficient since they were not evaluated by a second clinician, and thus, there were no areas of allodynia to be re-identified. Of the remaining 22 patients, allodynia was present in $70.97 \%$ of the patients, and there was a high level of agreement between examiners.

The patient group with allodynia was comprised of five males and $17 \mathrm{fe}$ -

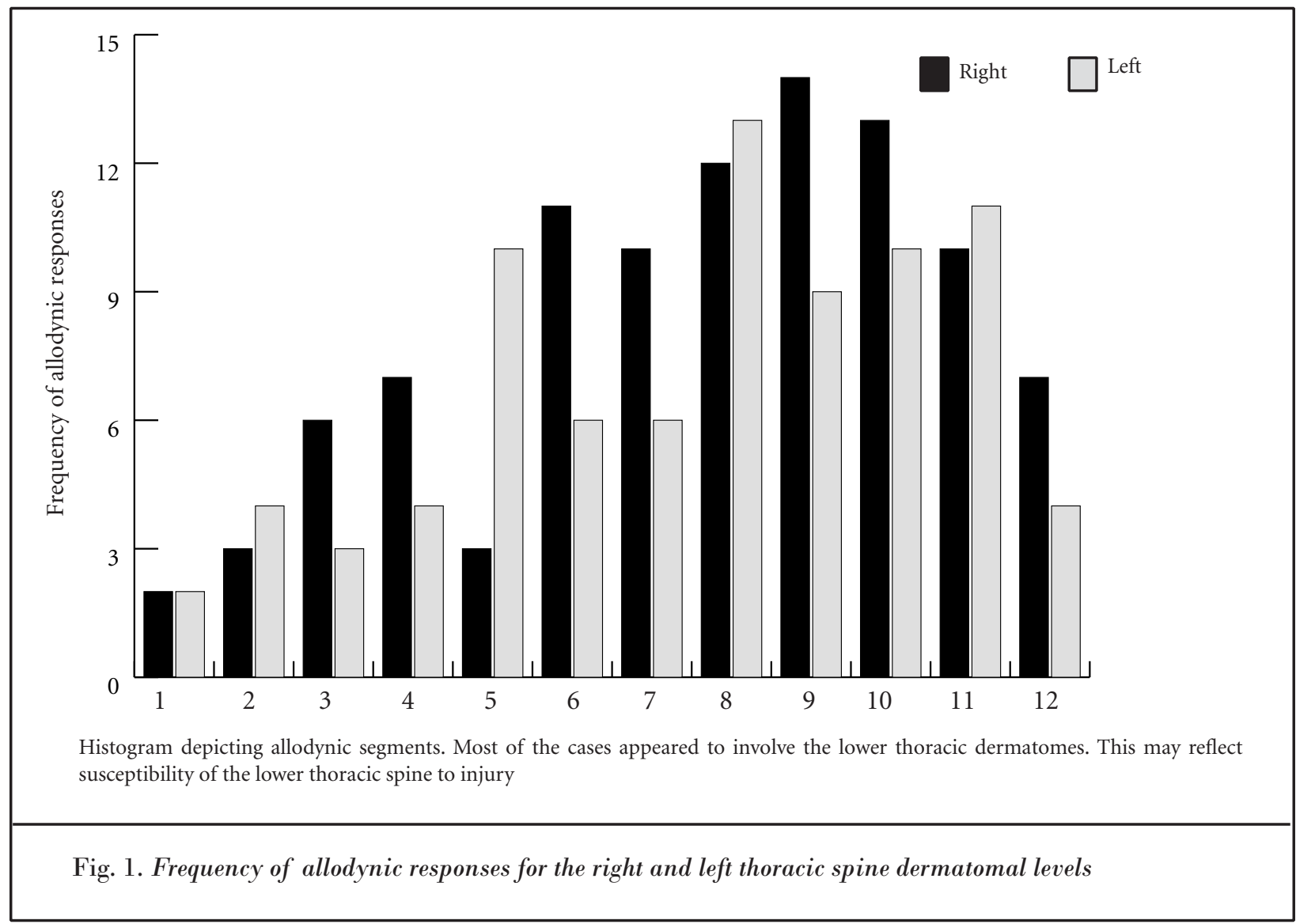

Pain Physician Vol. 8, No. 3, 2005 
males. Patient ages ranged from 16 to 72 years (mean age and standard deviation of 40.9 years $+/-14.8(\mathrm{n}=22)$. Average and standard deviation for male patients with allodynia was 52.0 years $+/-8.9(\mathrm{n}=5)$; the mean and standard deviation for female patients was 37.7 years $+/$ - 14.7 (n $=17$ ). The frequency distribution of allodynic dermatomes suggests that the lower thoracic segments were more commonly involved (Figure 1).

Table 1 contains the frequencies of how often certain agreement or disagreement occurred. There were four possible choices for agreement/disagreement: 1) both clinicians completely agreed that a visual response was present; 2) both clinicians completely disagreed; 3) clinician 1 agreed and clinician 2 disagreed; or 4) clinician 1 disagreed and clinician 2 agreed. The kappa coefficient is equal to 1 when there is complete agreement; the kappa coefficient is equal to 1 if, and only if, numbers off the diagonal in the 2 by 2 table (Table 1) are equal to 0 . The kappa coefficient is equal to 0 when complete disagreement occurred. The kappa coefficient indicated strong agreement between the clinicians $($ Kappa $=.8039)(\mathrm{Ta}-$ ble 2). The confidence interval (CI) for kappa was calculated using a level of significance 0.05 ( $95 \%$ CI, 0.7465 to 0.8613 ; $\mathrm{P}<0.05)$. The interval does not include 0 , which allows the null hypothesis to be rejected (e.g. that kappa is equal to 0 , or that clinicians completely disagree).

\section{Discussion}

This prospective study was an initial attempt to confirm the presence of allodynia and to quantify interrater reliability of the examination results in a whiplash cohort. We found that allodynia was present in about $70 \%$ of whiplash patients, and that findings were reproducible between different examiners, thus establishing interrater reliability. The results of this study contribute to the increasing ev-

Table 1: Two by two table containing frequency of agreement/disagreement

\begin{tabular}{|l|c|c|}
\hline \multirow{2}{*}{ Clinician 1 } & \multicolumn{2}{|c|}{ Clinician 2 } \\
\cline { 2 - 3 } Observed response (+) & Observed response (+) & Did not observe response (-) \\
\hline Did not observe response (-) & 123 & 17 \\
\hline Kappa coefficient of agreement & 24 & 364 \\
\hline
\end{tabular}

found that compared to controls, a whiplash group had significantly lower pain thresholds at both sites, suggesting that the hypersensitivity observed in healthy tissue results from alterations in the central processing of sensory stimuli. Furthermore, psychophysical studies $(8,9)$ also suggest the central mechanisms are responsible for ongoing pain in at least some whiplash patients.

Though further investigation into the mechanisms underlying allodynia in whiplash patients is warranted, some focus should be placed on identifying allodynia as reliable physical findings. Other hallmark findings consistent with whiplash injuries have been studied. The myofascial trigger point has been established as an interrater reliable physical finding when a training period for examiners is utilized $(11,12)$. It has also been demonstrated that thoracic tenderness relative to the cervical spine is not a normal finding in asymptomatic subjects (13). Indeed, the frequency distribution of allodynic dermatomes suggests that the lower thoracic segments are more commonly involved. Possible explanations include anatomic susceptibility to injury in the lower thoracic spine, thoracic kyphosis, or perhaps the construction of automobile seats. Assessment of passive intervertebral motion of the cervical spine demonstrates fair to moderate inter-examiner reliability (14).

This present study confirms that allodynia is a reproducible physical finding in whiplash patients. Presumably, due to exclusion criteria, the subjects examined in this study have no thoracic spine tissue damage, yet they did exhibit physical findings of allodynia on examination. Although cervical facet or discogenic pain generators and local myofascial trigger points in the thoracic paraspinal musculature may contribute to thoracic pain after whiplash, absence of objective exam findings consistent with tissue damage is common in patients with neck pain after whiplash (15). Nonetheless, the present observations suggest that central hypersensitivity may explain exaggerated pain after innocuous sensory input in whiplash-injured patients.

\section{CONCLUSION}

This prospective case series is an initial attempt to demonstrate the presence of allodynia as a physical finding in whiplash patients. Although the results of this 
case series may contribute to the hypothesis of an underlying central hypersensitivity mechanism sustaining chronic pain in whiplash patients, further investigation encompassing a non-whiplash cohort should be considered, and is underway. To enhance interrater reliability, questions of whether or not thoracic allodynia is present in other pain patient populations may be investigated. Such studies will contribute to the understanding of mechanisms involved in maintaining chronic pain in whiplash-injured patients

\section{Author Affiliation:}

Shannon L Bock, MMS, PA-C, CMT

11080 Circle Point Rd, Bldg. 2,

Suite 140

Westminster, CO 80020

E-mail: sbock@centenoclinic.com

Christopher J. Centeno, MD

Medical Director, Centeno Clinic 10080 Circle Point Rd., Bldg. 2

Suite 140

Westminster, CO 80020

E-mail: centenooffice@centenoclinic.com

James M. Elliott, MSPT

10080 Circle Point Rd.

Bldg. 2, Suite 140

Westminster, CO 80020

E-mail: jelliott@centenoclinic.com

\section{REFERENCES}

1. Koelbaek Johansen M, Graven-Nielsen T, Schou Olesen A, Arendt-Nielsen L. Generalised muscular hyperalgesia in chronic whiplash syndrome. Pain 1999; 83: 229234 .

2. Zimmerman M. Pathobiology of neuropathic pain. Eur J Pharmacol 2001; 429:2337.

3. Pitcher GM, Ritchie J, Henry JL. Nerve constriction in the rat: model of neuropathic, surgical and central pain. Pain 1999; 83: 37-46.

4. Ma QP, Woolf CJ. Progressive tactile hypersensitivity: An inflammation-induced incremental increase in the excitability of the spinal cord. Pain 1996; 67:97-106.

5. LaBuda Cl, Cutler TD, Dougherty PM, Fuchs PN. Mechanical and thermal hypersensitivity develops following kainate lesion of the ventral posterior lateral thalamus in rats. Neurosci Lett 2000; 290:7983.

6. Bulka A, Hao JX, Wiesenfeld-Hallin Z. Response characteristics of cutaneous mechanoreceptors in neuropathic rats. Neurosci Lett 2002; 317:89-92.

7. Curatolo $\mathrm{M}$, Petersen-Felix S, ArendtNielsen L, Giani C, Zbinden AM, Radanov BP. Central hypersensitivity in chronic pain after whiplash injury. Clin J Pain 2001; 17: 306-315.

8. Moog M, Quintner J, Hall T, Zusman M. The late whiplash syndrome: A psychophysical study. Eur J Pain 2002; 6:283-294.

9. Sheather-Reid RB, Cohen ML. Psychophysical evidence for a neuropathic component of chronic neck pain. Pain 1998; 75:341-347.

10. Sterling M, Treleaven J, Jull G. Responses to a clinical test of mechanical provocation of nerve tissue in whiplash associated disorder. Man Ther 2002; 7:89-94.

11. Gerwin RD, Shannon S, Hong CZ, Hubbard $D$, Gevirtz R. Interrater reliability in myofascial trigger point examination. Pain 1997; 69:65-73.

12. Sciotti VM, Mittak VL, DiMarco L, Ford LM, Plezbert J, Santipadri E, Wigglesworth J, Ball K. Clinical precision of myofascial trigger point location in the trapezius muscle. Pain 2001; 93:259-266.

13. Keating L, Lubke C, Powell V, Young T, Souvlis T, Jull G. Mid-thoracic tenderness: A comparison of pressure pain threshold between spinal regions, in asymptomatic subjects. Man Ther 2001; 6:34-39.

14. Smedmark V, Wallin M, Arvidsson I. Interexaminer reliability in assessing passive intervertebral motion in the cervical spine. Man Ther 2000; 5:97-101.

15. Radanov BP, Sturzenegger M, Di Stefano G. Long-term outcome after whiplash injury. A 2-year follow-up considering features of injury mechanism and somatic, radiologic, and psychosocial findings. Medicine 1995; 74:281-297 